\title{
Ultrasound elastography applications
}

\begin{abstract}
Rahman $M^{1}$, Islam $M^{2}$, Nargis $M^{3}$, Sarker $S^{4}$, Hasan $M J^{5}$, Quddush $R^{6}$, Sen $S^{7}$
Abstract

Ultrasound elastography (EUS) is a method to assess the mechanical properties of tissue, by applying stress and detecting tissue displacement using ultrasound. There are several EUS techniques used in clinical practice; strain (compression) EUS is the most common technique that allows real-time visualisation of the elastographic map on the screen. There is increasing evidence that EUS can be used to measure the mechanical properties of musculoskeletal tissue in clinical practice, with the future potential for early diagnosis to both guide and monitor therapy. This review describes the various EUS techniques available for clinical use, presents the published evidence on musculoskeletal applications of EUS and discusses the technical issues, limitations and future perspectives of this method in the assessment of the musculoskeletal system. Ultrasound elastography (EUS) is a recently developed ultrasound-based method, which allows the qualitative visual or quantitative measurements of the mechanical properties of tissue ${ }^{1}$. The technique was first introduced in vitro in the early 1990s, and subsequently evolved into a real-time tool for in vivo imaging of the distribution of tissue strain and elastic modulus ${ }^{2}$. EUS provides information on tissue stiffness, which complements and is independent from the acoustic impedance and vascular flow information provided by $\mathrm{B}$ mode and Doppler imaging, thus opening a new dimension in diagnostic imaging ${ }^{3}$. EUS is based upon the general principle that stress applied to tissue causes changes within it, which depend on the elastic properties of tissue ${ }^{3}$. Over the years of research on elasticity, there have been several approaches of EUS, resulting in different methods, depending on the way of tissue stress application and the used method to detect and construct an image of tissue displacement ${ }^{3}$. Strain (compression) EUS is the commonest technique that allows real-time visualisation of the image on the screen, and it has been successfully employed to detect and characterise lesions in a variety of tissues and organs ${ }^{5}$. Disease in the musculoskeletal system results in alterations to its biomechanical properties. Although EUS techniques have been extensively employed for in vitro research of muscle and tendon biomechanics since the early $1990 \mathrm{~s}^{6}$, the recent introduction of EUS into commercially available ultrasound systems has driven research activity towards potential clinical applications of this novel method in the musculoskeletal system ${ }^{7}$.

This review aims to describe the various EUS techniques available for clinical use, present the available published evidence on musculoskeletal applications of EUS, and finally discuss the limitations and future perspectives of this technique for assessing the musculoskeletal system.
\end{abstract}

Key word: Ultrasound elastography.

CBMJ 2013 Jan: Vol-02, No-01: P: 76-85

\section{Ultrasound elastography techniques}

There are several EUS techniques depending on the type of stress application and the method used to detect tissue displacement and construct the image ${ }^{3}$. The main techniques used in the clinical practice include strain EUS, shear wave EUS, transient EUS and acoustic radiation force EUS ${ }^{2}$. Each method is referred to in the literature and in commercially available systems, using a variety of different names, resulting in some confusion. The most commonly used method is strain EUS, also described as compression elastography, sonoelastography and real-time elastography ${ }^{8}$.
1. * Dr. Md. Masudur Rahman, Assoc Professor \& Head, Radiology and Imaging Community Based Medical college Bangladesh.

2. Dr. Mahzabeen Islam Assistant Professor, Radiology and Imaging Community Based Medical college Bangladesh.

3. Dr. Mahbuba Nargis Senior Consulatant, Maternity Hospital Azimpur, Dhaka.Bangladesh.

4. Dr. Samar Chandra Sarker Associate Professor, Radiology and Imaging Enam Medical College, Bangladesh.

5. Dr. Mahmud Javed Hasan Assistant Professor Nephrology Community Based Medical college Bangladesh.

6. Dr. ASM Ruhul Quddush

Assistant Professor Pediatrics Community Based Medical college Bangladesh.

7. Dr. Shila Sen

Professor \& Head, Obstetrics \& Gynecology Community Based Medical college Bangladesh.

* Address of correspondence:

E-mail:drmasud64@gmail.com

Mobile : + 8801732398297 
The technique is based on low-frequency compression of the tissue, which is usually applied manually via the hand-held ultrasound transducer (free-hand EUS) ${ }^{8}$, or in some cases using physiological body movement such as respiration or pulsation ${ }^{5}$. The main principle of strain EUS is that a compressive force is applied to tissue causing axial tissue displacement (strain), which is then calculated by comparing the echo sets before and after the compression ${ }^{2}$. Strain EUS is based upon Hooke's law for the calculation of Young's elastic modulus (E), a physical quantity measuring stiffness. By assuming that the applied stress is uniform, the elastic moduli are inversely proportional to the measured strain (E5stress/ strain). Strain is the change in size or shape produced by a system of forces, and it is expressed as a ratio (e.g. the change in length per unit length). The force acting on unit area is known as the stress ${ }^{1}$. Strain EUS is actually measuring the relative strain of one area vs another, and displaying it as a map. Because manual compression is used, there may be differences in displacement depending on different levels of pressure, different tissue depths, correct probe alignment and out-ofplane movements of the transducer, resulting in considerable operator dependence. Sophisticated methods of oversampling are used to improve signal-to-noise ratio and reduce artefacts in order to reduce the effect of some of the above limitations ${ }^{1}$. This technique allows direct visualisation of the strain information superimposed on the conventional B-mode image as a strain distribution map ("the elastogram"), which, for visualisation purposes, is greyscale or colour coded, and displayed next to the B-mode image on the screen (Figure 1). The grey or colour scale encoding is chosen by the user. Most often red is used for encoding soft tissues, blue for hard tissues and yellow/green for tissue of intermediate stiffness. The elasticity information derived by this method is qualitative or semi-quantitative ${ }^{3}$. The strain of each area is compared with the remaining tissue within the elastogram, so the elastogram is a relative image available for visual comparison only. The semiquantitative measurement method includes the ratio of the relative strains between the area of interest and a reference area (usually fat). Strain EUS is mainly applied in the field of oncology imaging to detect and differentiate malignancy in breast, prostate, liver, pancreas, thyroid, cervix and lymph nodes ${ }^{9}$. Strain EUS is also the technique mainly employed so far for musculoskeletal applications $^{7}$.

Acoustic radiation force impulse (ARFI) is a type of strain EUS whereby tissue is excited internally by a focused ultrasound pulse, instead of external (manual or physiological) compression ${ }^{7}$. As the ultrasound pulse travels through the tissue, soft tissue experiences larger displacement than hard tissue. After the excitation and displacement by the pulse, the tissue relaxes to its original configuration. The tissue displacement by the original push pulse can be measured using the application of several short-time pulse echoes, which provides data for comparison with the reference image ${ }^{7}$. The technique also results in a qualitative colour-coded or greyscale elastogram depicting relative tissue stiffness. This method has the advantage of imaging deeper tissue, not accessible by superficial external compression and has been used mainly for liver, thyroid and breast imaging ${ }^{7}$.

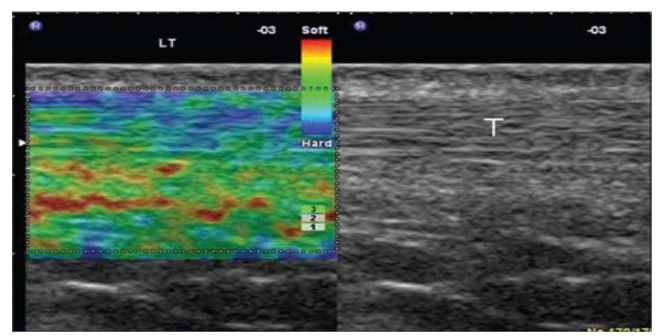

A

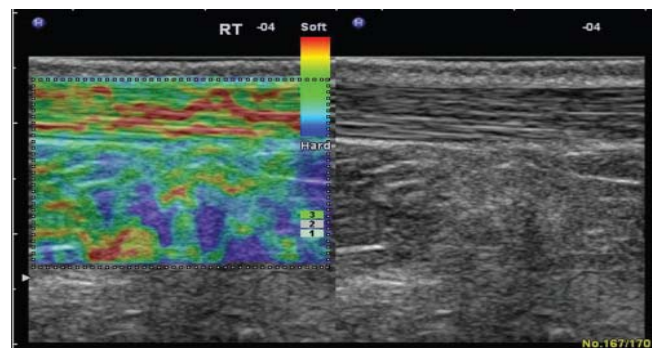

B 

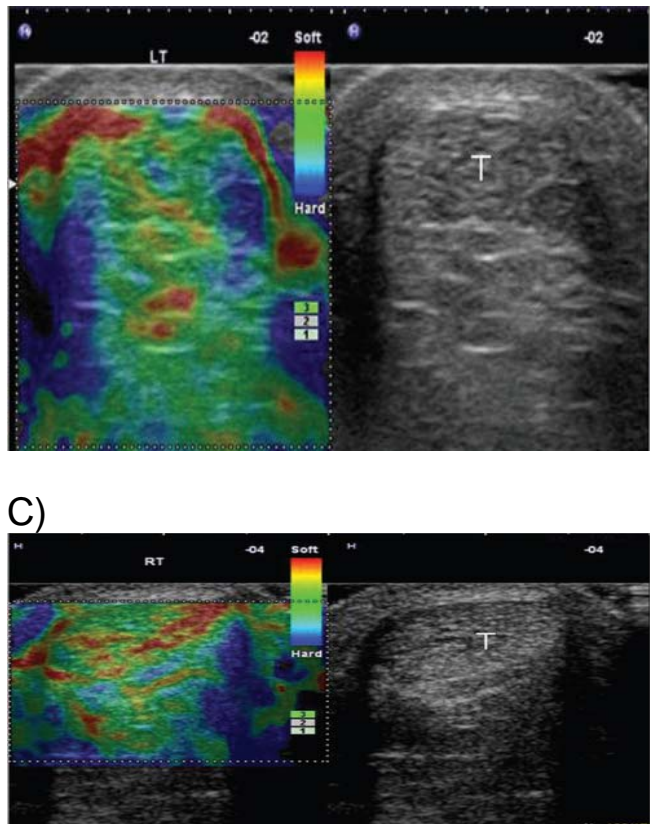

D)

Figure 1. (a, b) Longitudinal and (c, d) transverse free-hand strain elastograms of the middle third of asymptomatic Achilles tendons ( $T$ ) showing the two distinct ultrasound elastography patterns of normal tendons. Type $1(\mathrm{a}, \mathrm{c})$ tendons appear homogeneously stiff, (green/blue) with no distinct soft (red) areas. Type 2 (b, d) tendons appear considerably inhomogeneous with soft (red) areas (longitudinal bands or spots), which did not correspond to any changes in B-mode ultrasound. The retro- Achilles fat appears as a mosaic of green, red and blue. Note the red areas at the lateral and medial sides of the tendon in the transverse plane (c, d), corresponding to an artefact, secondary to difficulty in stabilising the hand-held transducer.

Shear wave EUS is based on a completely different physical principle. Shear waves are generated within tissue when the conventional ultrasound waves produced by the transducer interact with tissue ${ }^{10}$. Shear waves propagate perpendicular to the axial displacement caused by the ultrasound pulse and attenuate approximately 10000 times more rapidly than conventional ultrasound ${ }^{10}$.
By use of ultrafast algorithms, the velocity of shear waves can be measured and used to evaluate tissue stiffness by calculating the elastic Young's modulus according to the formula: E<36V2 (E5Young's modulus in kPa, V5shear wave velocity in cms-1) ${ }^{10}$. This technique results in both qualitative colour coded elastograms and also quantitative maps either of elasticity (in $\mathrm{kPa}$ ) or of shear wave velocity (in cms-1). This method is more objective than strain EUS, because of the lack of tissue compression, the direct assessment of elasticity and the quantitative measurements provided. However, there are concerns about the use of this method in very superficial structures, as a certain depth of ultrasound penetration is needed for shear waves to be produced ${ }^{10}$.

Transient EUS, also known as vibrationcontrolled elastography, is a variant of shear wave EUS, whereby the external compression is applied by using a short-tone burst of vibration ${ }^{11}$. The method also relies on the estimation of the velocity of shear waves in tissue, but in order to avoid the bias caused by reflections and interferences occurring between the tissues, vibration is transient, so that forward waves can be separated from the reflected waves 11 . Transient EUS is mainly used in examinations for liver disease ${ }^{11}$.

Ultrasound elastography for the examination of tendons

The Achilles tendon has provided most of the clinical data available so far in musculoskeletal applications; it was the first area to be investigated using free-hand strain EUS (Table 1). In a study of 50 asymptomatic and sonographically normal Achilles tendons in healthy volunteers, the normal tendons were found to have two distinct EUS patterns (Figure 1) 12 . They were either homogeneously hard structures or, in the majority of cases (62\%), they were found to have considerable inhomogeneity with soft areas (longitudinal bands or spots), which did not correspond to any changes in Bmode or Doppler ultrasound ${ }^{12}$. These findings were confirmed in two studies by the same research group, comparing normal 
(asymptomatic) and abnormal (symptomatic) tendons ${ }^{8}$.

The asymptomatic tendons were found to be homogeneously hard in $86-93 \%$ of cases, containing mild softening (yellow) in $7-12 \%$ of cases and containing marked softening (red) in $0-1.3 \%$ of cases $^{8}$. By contrast, symptomatic tendons were found in EUS to contain marked softening in 57\%, mild softening in $11 \%$ and no soft areas (hard structures) in $32 \%$ of cases ${ }^{5}$. The alterations in asymptomatic tendons were mainly observed in the tendon mid-portion and were not always found to correspond to alterations in conventional ultrasound ${ }^{8}$. Mild softening (yellow) was not correlated with conventional ultrasound abnormalities, whereas marked softening (red) was found mainly in cases with ultrasound disease, so the authors suggest that only marked soft areas should be considered as abnormal in Achilles tendon EUS $^{8}$ (Figure 2). The nature of the EUS alterations found in asymptomatic and sonographically normal tendons is not yet completely understood; it is suggested that they may either correspond to early (preclinical) changes not yet evident using ultrasound or to false-positive findings, secondary to tissue shifting/non-axial movement at interfaces between collagen fibres ${ }^{8}$. To date no histopathology or followup studies are available to elucidate the above presumptions. However, another study used strain EUS to assess 12 patients with Achilles tendinopathy using ultrasound and MRI and found increased stiffness in the abnormal tendons, compared with the nonsymptomatic, which were softer ${ }^{14}$. These findings are completely different from those previously reported and emphasise the need for further research on EUS of the Achilles tendon. The accuracy of free-hand strain EUS vs clinical examination and conventional ultrasound, as well as the reproducibility of EUS for the Achilles tendon, has been assessed by the above studies ${ }^{18}$. The overall correlation between ultrasound and EUS findings was found to be excellent (accuracy 97\%), provided that mild softening (yellow) is considered physiological and only distinct red areas are considered abnormal ${ }^{19}$

. Compared to clinical findings, EUS had a mean sensitivity of $93.7 \%$ and a specificity of $99.2 \%^{8}$. The inter- and intra-observer reproducibility of the EUS has been measured for normal and abnormal Achilles tendons by two studies, and was found to be good to excellent if the assessment of the Achilles tendon elastogram was performed qualitatively, using the predominant colour pattern ${ }^{14}$, and poor (variation 29-37\%) if semi-quantitative software measurements (strain ratio) were employed ${ }^{12}$. Besides Achilles tendon disease, lateral epicondylitis has also been studied in a single publication using EUS, which showed significant softening in the abnormal extensor tendons with strong correlation with ultrasound and clinical findings ${ }^{7}$. EUS localised more focal lesions and more cases of collateral ligament and fascia involvement than ultrasound and thus had greater accuracy than greyscale ultrasound by comparison with clinical examination as the reference standard ${ }^{7}$. Preliminary data presented in meetings has also suggested the potential role of strain EUS for the assessment of a variety of traumatic and degenerative disorders of muscles and tendons ${ }^{15}$; however, there are to date no case-controlled published studies available for these applications. Although there are studies on shear wave/transient EUS for pre-clinical examination of tendon and muscle elasticity ${ }^{16}$, this technique has not been widely used for musculotendinous disease in clinical practice. There is only one study measuring the mean elasticity values of masseter, gastrocnemius and supraspinatus muscles as well as the Achilles tendon in normal volunteers, in order to set the normal values for comparison with abnormal conditions $^{17}$. 
Review Article

Table 1. Summary of EUS applications for tendons

\begin{tabular}{|c|c|c|c|c|}
\hline Article & Correlation with & Area examined & Number of cases & Results regarding EUS \\
\hline $\begin{array}{l}\text { Drakonaki et } \\
\text { al, } 2009 \text { [17] }\end{array}$ & $\begin{array}{l}\text { Conventional } \\
\text { ultrasound }\end{array}$ & Achilles & $\begin{array}{c}50 \text { normal } \\
\text { tendons ( } 25 \\
\text { volunteers) }\end{array}$ & $\begin{array}{l}\text { Two normal patterns } \\
\text { Reproducibility better in } \\
\text { longitudinal plane }\end{array}$ \\
\hline $\begin{array}{l}\text { De Zordo et } \\
\text { al, } 2010 \text { [18] }\end{array}$ & $\begin{array}{l}\text { Conventional } \\
\text { ultrasound }\end{array}$ & Achilles & $\begin{array}{l}50 \text { normal } \\
\text { tendons ( } 25 \\
\text { volunteers) }\end{array}$ & $\begin{array}{l}\text { Accuracy } 97 \% \\
\text { Good correlation with } \\
\text { ultrasound } \\
\text { Two patterns (grades) in } \\
\text { normal tendons } \\
\text { Three patterns (grades) in } \\
\text { symptomatic tendons }\end{array}$ \\
\hline $\begin{array}{l}\text { De Zordo et } \\
\text { al, } 2009 \text { [19] }\end{array}$ & $\begin{array}{l}\text { Clinical exam } \\
\text { Conventional } \\
\text { ultrasound }\end{array}$ & Achilles & $\begin{array}{l}80 \text { normal } \\
\text { tendons (40 } \\
\text { volunteers) }\end{array}$ & $\begin{array}{l}\text { Good correlation with } \\
\text { ultrasound } \\
\text { Three patterns (grades) in } \\
\text { normal tendons }\end{array}$ \\
\hline $\begin{array}{l}\text { De } \\
\text { Sconfienza } \\
\text { et al, 2010 } \\
{[21]}\end{array}$ & $\begin{array}{l}\text { Clinical exam } \\
\text { Conventional } \\
\text { Ultrasound } \\
\text { MR }\end{array}$ & Achilles & $\begin{array}{l}12 \text { tendons (12 } \\
\text { patients) } \\
36 \text { normal } \\
\text { tendons (18 } \\
\text { volunteers) }\end{array}$ & $\begin{array}{l}\text { Good correlation with MRI } \\
\text { Symptomatic tendons are } \\
\text { harder }\end{array}$ \\
\hline $\begin{array}{l}\text { De Zordo et } \\
\text { al, } 2009 \text { [22] }\end{array}$ & $\begin{array}{l}\text { Clinical exam } \\
\text { (VAS score) } \\
\text { Conventional } \\
\text { ultrasound }\end{array}$ & $\begin{array}{l}\text { Common } \\
\text { Extensor } \\
\text { tendons } \\
\text { (elbow) }\end{array}$ & $\begin{array}{l}38 \text { tendons (32 } \\
\text { patients) } \\
44 \text { normal } \\
\text { tendons ( } 28 \\
\text { volunteers) }\end{array}$ & $\begin{array}{l}\text { Accuracy } 94 \% \\
\text { Good correlation with } \\
\text { ultrasound } \\
\text { Greater sensitivity and } \\
\text { accuracy than ultrasound }\end{array}$ \\
\hline
\end{tabular}

EUS, ultrasound elastography; VAS, visual analogue scale.
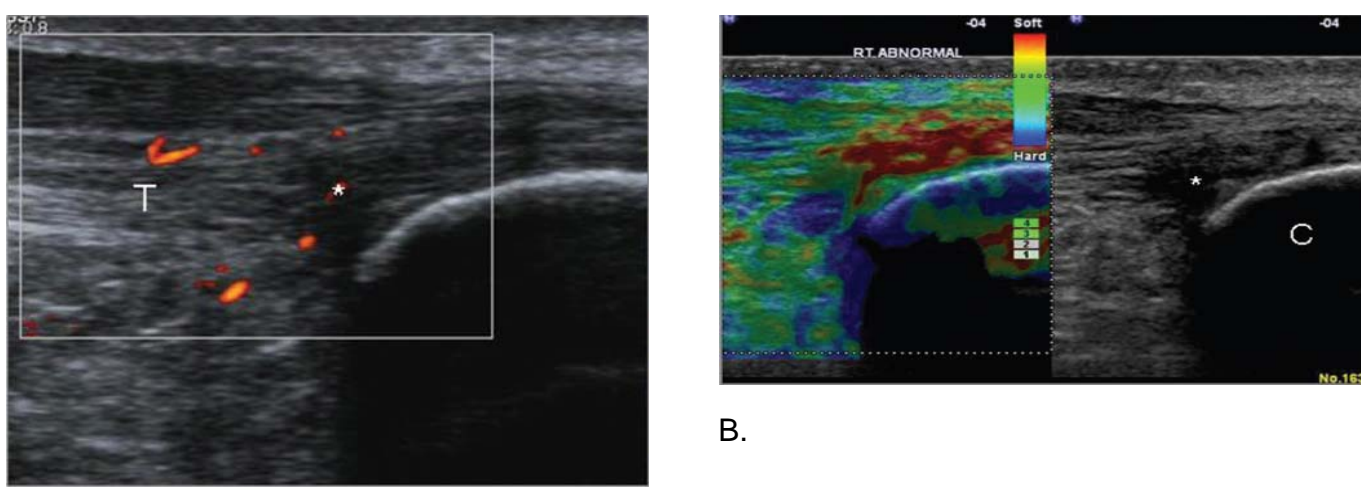

B.

A. 
Review Article

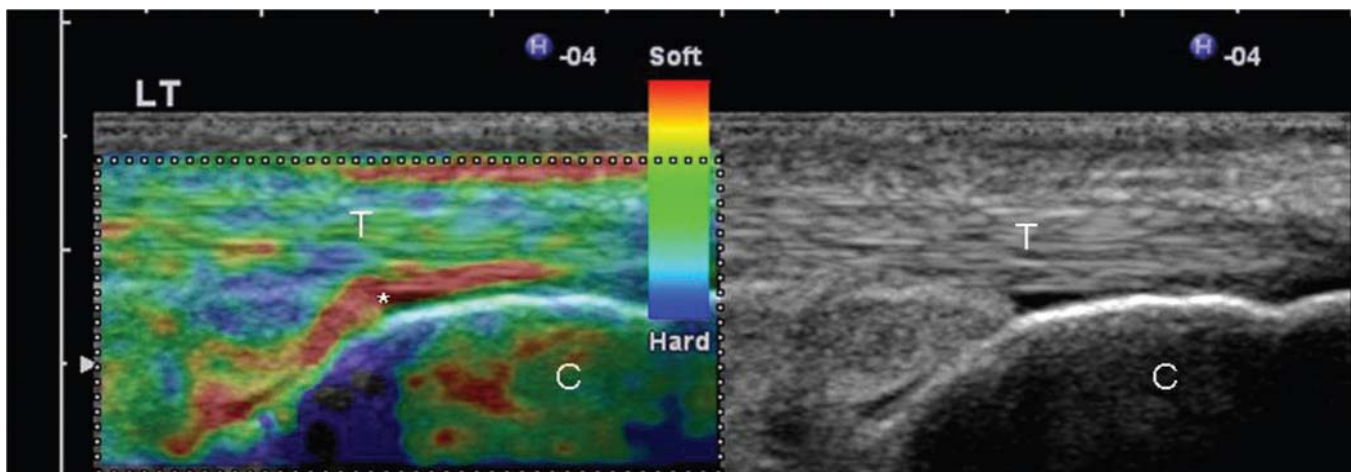

C.

Figure 2. (a) Longitudinal colour Doppler ultrasound image and (b) free-hand strain elastogram of a 23-year-old recreational runner with insertional Achilles tendinopathy. There is hypoechogenicity and neovascularity at the tendinopathic area [asterisk in (a)], which appears softer (red) compared with the stiff (blue/green) normal-appearing remaining tendon $(T)$. The retro- Achilles fat appears as a mosaic of various levels of stiffness. (c) The elastogram of a normal (asymptomatic) Achilles tendon calcaneal insertion is presented for comparison. The retrocalcaneal bursa (asterisk) appears considerably softer (red) compared with the stiffer tendon (green/yellow). Note the presence of soft (red) foci beneath the calcaneal bone (C), which correspond to artefacts. LT, left.

EUS helped in a hallmark of polymyalgia rheumatica, from the stiffer supraspinatus tendon, which was not possible in B-mode ultrasound, suggesting the use of EUS for localisation of soft tissue damage ${ }^{20}$. Another study in patients with systemic sclerosis showed the EUS could assess skin involvement at the forearm, where thickening of the dermis was evident, but was not useful for assessing finger involvement, where the bone hyper-reflection was a limiting factor ${ }^{11}$. A case series has assessed EUS for the differentiation of rheumatoid nodules and tophi, showing that rheumatoid nodules were significantly less elastic than tophi and suggesting a possible role of this method for the investigation of soft tissue nodules ${ }^{21}$. In a pictorial assay of EUS applications, inflammatory synovitis (rheumatoid arthritis) was shown to be of intermediate stiffness,

Infectious synovitis (due to tuberculosis) was softer, whereas fatty villous proliferations (lipoma abrorescens) and pigmented villonodular synovitis were predominantly soft, as opposed to synovial sarcoma, which was hard ${ }^{15}$. There is only one study on the use of EUS for the examination of common soft tissue neck masses, the nature of which was confirmed with biopsy or further imaging ${ }^{7}$. The study showed that lipomata and venous/lymphatic vascular malformations and thyroglossal cysts were predominantly soft, which was attributed to their fat/liquid content, and neurogenic tumours and dermoid/sebaceous cysts were stiffer, whereas abscesses displayed various levels of stiffness ranging from soft to intermediate, presumably due to various liquid and solid components ${ }^{7}$. Although there was considerable overlap in EUS scores, the authors suggest the potential role of EUS in cases of equivocal B-mode findings, for guiding aspiration or biopsy in abscesses or mixed solid/cystic lesions 22. Most of the above data are based on non-controlled studies with a small number of cases and even the authors agree that, although promising, further investigation is needed to determine the possible role of EUS in the diagnostic work-up of rheumatic diseases ${ }^{7}$. Technical considerations and limitations of ultrasound elastography

The major problem in the application of EUS is that there are a wide variety of techniques and processing algorithms currently available for producing and displaying elastographic images and therefore the findings as well as the artefacts or limitations may be highly dependent on the technique and may be specific to a specific system. Experience regarding technical problems and the means 
of resolving them has resulted from the use of free-hand compression EUS. Compression EUS is technically very challenging in terms of the proper application of the technique. It is difficult to produce

high quality, artefact-free cine loops of decompression- compression cycles. The problems are associated with either inherent limitations of the technique itself or the characteristics of the musculoskeletal system. A major issue associated with compression EUS is determining the correct amount of pressure to be applied on tissue. The pressure should be moderate, described as the level of pressure that maintains contact with skin and for which the association between pressure and strain is proportional ${ }^{5}$. Very high or low pressure should be avoided, as the elastic properties of tissue become non- linear ${ }^{23}$. Most EUS systems now provide software, which allows a feedback of the amount of pressure as a visual indicator/bar displayed on the screen alongside sonographic images thus ensuring the correct application of pressure. To minimise intra-observer variation and avoid transient temporal fluctuations, the scoring or measurements in the elastograms should be based on examination of entire cine loops instead of single static images ${ }^{12}$. The most common method to assess the elastograms is by viewing representative images derived from cine loops of at least three compression- relaxation cycles ${ }^{8}$. The images should be chosen at the compression phase and in the middle of each cycle, as the calculation of the elastogram at the initial and final stages of each cycle will be

inaccurate ${ }^{8}$. Another major problem in strain EUS is the lack of quantitative measurements. This has led researchers to use various methods for the assessment of the elastograms, which include semiquantitative measurements (strain ratio) ${ }^{12}$, qualitative assessment visual assessment of elastograms using patterns, scores or grades 8 , or by using commercially available external computer software ${ }^{7}$. This has led to considerable confusion in the interpretation of the findings, a lack of reproducibility and difficulty in comparing the results from different studies, even if the same technique (strain EUS) is applied in all cases. When using EUS for examining musculoskeletal tissue, special issues should be taken into consideration. In conventional musculoskeletal ultrasound the amount of pressure should be as light as possible, so as not to distort the underlying tissues (e.g. fluid within bursa or synovial cavity), whereas in EUS a certain amount of pressure is necessary to allow the correct application of the technique. The examination probe should always be held perpendicular to the tissue to avoid anisotropy, as the B-mode appearance influences the acquisition of EUS data ${ }^{8}$. Although tendon images should be taken in both transverse and longitudinal planes, longitudinal images are of better quality, as it has been shown that the reproducibility of transverse images of the Achilles tendon is less than that of longitudinal images because of artefacts at the medial and lateral sides of the image secondary to unilateral pressure and out-of-plane movements of the transducer ${ }^{12}$. There are elasticity changes at the borders of the elastogram attributed to inhomogeneous application of pressure ${ }^{8}$, and so overlapping images should be acquired to overcome this problem. There are also limitations and difficulties related to the anatomy of the area examined. EUS is especially problematic in cases of superficial protuberant masses and in areas with prominent adjacent bony structures (e.g. at the level of the malleoli when examining tibialis posterior and the peroneal tendons), where it is difficult to apply uniform compression over the entire region of interest 7 . Another important parameter is the size of the elastogram. The elastogram displays the elasticity of each tissue relative to the remaining tissue within it. Therefore, the amount and level of stiffness of the surrounding tissue influences the appearance of the tissue of interest. This is not a major problem in tissues such as the breast where the surrounding tissue is fairly homogeneous (fat and glandular tissue). In musculoskeletal EUS, however, the elastogram may include tissues with wide elasticity differences (fat, tendon, bone, muscle), leading to a wider scatter in the acquired elasticity data. For the Achilles tendon, the suggested standard size for longitudinal scans is a depth of three times the tendon and about three quarters of the screen, and for transverse scans the paratenon should be included ${ }^{8}$. However, 
Review Article

this suggestion is not universally applied, leading to difficulties in comparisons between studies. Another standardisation problem is the distance between the probe and the tissue of interest.

In many musculoskeletal applications, the tissue of interest is very superficial or even lies directly under the skin (e.g.Achilles tendon). In most ultrasound systems a minimum distance (usually $1.2 \mathrm{~mm}$ ) from the skin is needed to place the box of the elastogram, so in thin people the use of gel pads or probe adaptors is necessary to increase the distance between the skin and probe ${ }^{24}$. Using these stand-off devices has been proven not to influence the appearance of the elastogram ${ }^{8}$. In conventional musculoskeletal imaging, the use of large amounts of gel is common practice in order to create an even surface and to reduce the amount of pressure on the tissue. However, when performing EUS for musculoskeletal applications, care should be taken not to include the gel in the box of the elastogram, as it results in dramatic changes, making the tendons appear considerably stiffer compared with the gel (Figure 3). Several artefacts can be encountered during the application of EUS in musculoskeletal tissues, which reduce the quality of the elastograms and may lead to misinterpretation of the images. These include fluctuant changes at the edges of the elastogram and at the medial and lateral borders of thin structures (such as the Achilles tendon in the axial plane) due to instability and out-of-plane movement of the transducer (Figure 1b) ${ }^{14}$. Occasionally red (soft) lines may appear around calcifications or phleboliths, behind dense bone and at the superficial margin of homogeneous lesions (such as lipomata; Figure 2b) 8 . Characteristic artefacts are also associated with cystic masses, which appear as a mosaic of all levels of stiffness (all colours), and with lesions adjacent to major vessels, where pulsations result in mistracking of echoes ${ }^{25}$. Familiarity with the above artefacts is important, as they should be excluded from the qualitative or quantitative scoring of the elastograms.
EUS probably represents the most important technical development in the field of ultrasonography since Doppler imaging. It has many advantages over other methods of tissue elasticity estimation, such as MR elastography, as it is a low-cost, fast, noninvasive system, and has the potential of wider clinical availability.

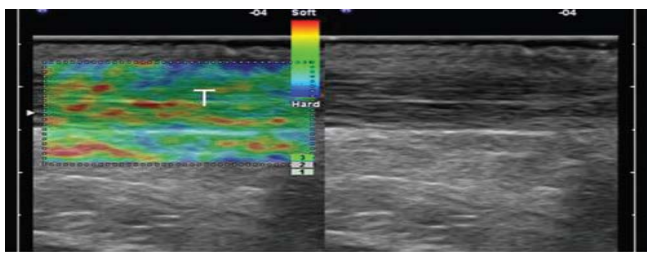

A.

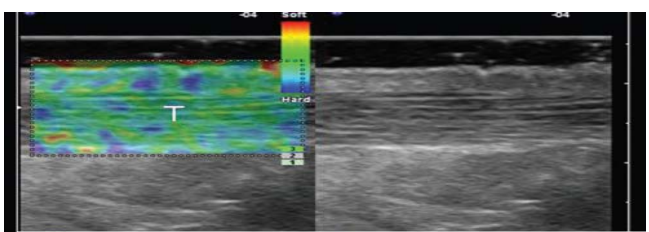

B.

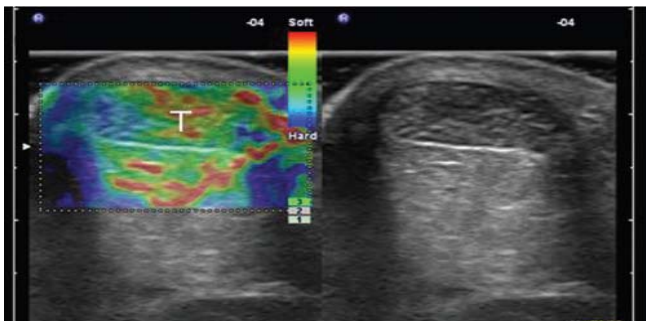

C.

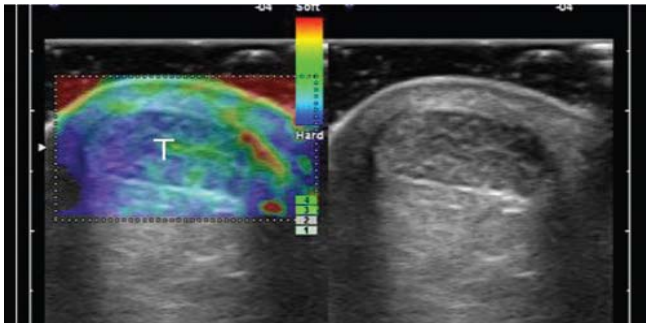

D.

Future perspectives 
Figure 3. The impact of gel on the strain elastograms. (a, b) Longitudinal and (c, d) axial elastograms of the same asymptomatic Achilles tendon $(T)$. The inclusion of a small amount of gel in the elastogram $(b, d)$ results in a homogeneously stiffer tendon without areas of distinct softening (red), which are evident when no gel is included $(a, c)$. The level of pressure and the ultrasound elastography settings were kept stable.

The evidence so far seems very promising that EUS can be used to assess the mechanical properties of musculoskeletal tissues in the clinical setting. Preliminary data show that EUS may even be more sensitive than MRI or grey-scale ultrasound in detecting sub clinical changes of muscle and tendon, and therefore could be valuable for early diagnosis and during rehabilitation medicine. EUS could be used as a research tool to provide insight into the biomechanics and pathophysiology of musculotendinous disease. However, despite the great interest in the technique, the published literature is still very limited and mainly depends on case reports or non-controlled studies with small study populations using various EUS techniques and scoring systems. There are several technical issues, including a lack of quantification methods, artefacts, limitations and variation in the application of the technique by different users, which limit the reproducibility of the method. There are doubts regarding the potential clinical utility of this new diagnostic tool, as in most cases the EUS showed changes already evident on conventional ultrasound or colour Doppler imaging, whereas EUS changes not evident on conventional imaging were occult, and therefore not clinically important. For all of the above reasons, we think that a more systematic and structured approach to the investigation of this new method should be undertaken. First, we advocate standardisation of EUS for soft tissue applications, based on the manufacturers' suggestions and consensus between users, employing parameters such as the size of the elastogram, the use of adaptors/pads/gel, the scoring systems and so on. This will be of paramount importance in achieving consistency in the application of the technique and should allow comparisons between studies. In order to overcome the technical issues associated with the use of EUS in superficial tissues, close collaboration between the industry and clinical researchers will allow the clinical experience to be used for the development of optimized protocols dedicated to musculoskeletal applications.

Second, we need to carefully establish the indications for EUS. These would ideally focus on the cohort of patients with symptomatic but non-ultrasound-evident disease, patients at risk or patients at very early stages of disease, in order to investigate whether EUS is more sensitive than conventional imaging in depicting earlier clinically important changes. Multicentre long-term controlled studies are needed; these should include large populations of different ages and levels of activity with longterm follow-up and correlation with histology, conventional imaging (ultrasound and MRI), and biomechanical and clinical data, in order to describe the pattern and nature of EUS findings and their clinical significance. Finally, newer algorithms that allow quantitative assessment of elasticity such as shear wave EUS or ARFI should be studied and compared with qualitative strain EUS.

\section{Conclusion}

Owing to lack of standardisation and limited research, EUS in its current form remains a highly subjective technique, with debatable clinical value.With the proper standardisation and further structured research, EUS may become a valuable supplementary tool in the investigation of musculoskeletal disease. 


\section{References}

1. Hall TJ. AAPM/RSNA physics tutorial for residents: topics in US: beyond the basics: elasticity imaging with US. Radiographics 2003;23:1657-71.

2. Ophir J, Cespedes I, Ponnekanti H, Yazdi Y, Li $X$. Elastography: a quantitative method for imaging the elasticity of biological tissues. Ultrason Imaging 1991;13:111-34.

3. Garra BS. Elastography: current status, future prospects, and making it work for you. Ultrasound Q 2011;27:177-86.

4. Garra BS. Elastography: current status, future prospects, and making it work for you. Ultrasound Q 2011;27:177-86.

5. Dighe $M$, Bae $U$, Richardson ML, Dubinsky TJ, Minoshima S, Kim Y. Differential diagnosis of thyroid nodules with US elastography using carotid artery pulsation. Radiology 2008;248:662-9.

6. Levinson SF, Shinagawa $M$, Sato $T$. Sonoelastic determination of human skeletal muscle elasticity. J Biomech 1995;28:1145-54.

7. Li Y, Snedeker JG. Elastography: modalityspecific approaches, clinical applications, and research horizons. Skeletal Radiol 2011;40:389-97

8. Park GY, Kwon DR. Application of real-time sonoelastography in musculoskeletal diseases related to physical medicine and rehabilitation. Am J Phys Med Rehabil 2011;in press. E-pub ahead of print 16 August 2011.

9. Thomas A, Ku"mmel S, Gemeinhardt $O$, Fischer T. Real-time sonoelastography of the cervix: tissue elasticity of the normal and abnormal cervix. Acad Radiol 2007;14:193200.

10. Bercoff J, Tanter M, Fink M. Supersonic shear imaging: a new technique for soft tissue elasticity mapping. IEEE Trans Ultrason Ferroelectr Freq ontrol 2004;51:396-409.

11. Sandrin $L$, Fourquet $B$, Hasquenoph JM, Yon $S$, Fournier $C$, Mal $F$, et al. Transient elastography: a new noninvasive method for assessment of hepatic fibrosis. Ultrasound Med Biol 2003;29:1705-13.

12. Drakonaki EE, Allen GM, Wilson DJ. Real-time ultrasound elastography of the normal Achilles tendon: reproducibility and pattern description. Clin Radiol 2009;64:1196-202.

13. Friedrich-Rust $M$, Ong MF, Herrmann E, Dries $V$, Samaras $P$, Zeuzem $S$, at al. Real-time elastography for noninvasive assessment of liver fibrosis in chronic viral hepatitis. AJR Am J Roentgenol 2007;188:758-64.

14. De Sconfienza $L M$, Silvestri E, Cimmino MA. Sonoelastography in the evaluation of painful Achilles tendon in amateur athletes. Clin Exp Rheumatol 2010;28: 373-8.
15. Lalitha $P$, Reddy MCh, Reddy KJ. Musculoskeletal applications of elastography: a pictorial essay of our initial experience. Korean J Radiol 2011;12:365-75.

16. Gennisson JL, Deffieux T, Mace' E, Montaldo $G$, Fink $M$, Tanter $M$. Viscoelastic and anisotropic mechanical properties of in vivo muscle tissue assessed by supersonic shear imaging. Ultrasound Med Biol 2010;36:789_ 801

17. Arda K, Ciledag N, Aktas E, Aribas BK, Ko" se $K$. Quantitative assessment of normal softtissue elasticity using shear-wave ultrasound elastography. AJR Am J Roentgenol 2011;197:532-6.

18. Sconfienza $L M$, Silvestri $E$, Bartolini $B$, Garlaschi G, Cimmino MA. Sonoelastography may help in the differential diagnosis between rheumatoid nodules and tophi. Clin Exp Rheumatol 2010;28:144-5.

19. Bhatia KS, Rasalkar DD, Lee $Y P$, Wong $K T$, King $A D$, Yuen $Y H$, et al. Real-time qualitative ultrasound elastography of miscellaneous nonnodal neck masses: applications and limitations. Ultrasound Med Biol 2010;36:164452.

20. Goertz RS, Zopf $Y$, Jugl V, Heide R, Janson $C$, Strobel $D$, et al. Measurement of liver elasticity with acoustic radiation force impulse (ARFI) technology: an alternative noninvasive method for staging liver fibrosis in viral hepatitis. Ultraschall Med 2010;31:151-5.

21. Tozaki M, Isobe S, Fukuma E. Preliminary study of ultrasonographic tissue quantification of the breast using the acoustic radiation force impulse (ARFI) technology. Eur J Radiol 2011;80:e182-7.

22. Friedrich-Rust M, Romenski O, Meyer G, Dauth $N$, Holzer K, Gru" nwald F, et al. Acoustic Radiation Force Impulse- Imaging for the evaluation of the thyroid gland: a limited patient feasibility study. Ultrasonics 2012;52:69-74.

23. Bercoff J, Tanter M, Fink M. Supersonic shear imaging: a new technique for soft tissue elasticity mapping. IEEE Trans Ultrason Ferroelectr Freq ontrol 2004;51:396-409.

24. Sandrin L, Fourquet B, Hasquenoph JM, Yon $S$, Fournier $C$, Mal $F$, et al. Transient elastography: a new noninvasive method for assessment of hepatic fibrosis. Ultrasound Med Biol 2003;29:1705-13.

25. Grainger AJ. Highlights of the European Society of Musculoskeletal Radiology (ESSR) annual meeting 2010. Skeletal Radiol 2011;40:137-9. 